\title{
STURMIAN SPIRALS IN LORENTZ-MINKOWSKI PLANE
}

\author{
KAZIM İLARSLAN, ALI UÇUM AND IVAÏLO M. MLADENOV
}

Presented by Ivaïlo M. Mladenov

Abstract. The Sturm spirals which can be introduced as those plane curves whose curvature radius is equal to the distance from the origin are embedded in to one parameter family of curves. In this paper, we consider the spacelike and timelike Sturmian spirals in Lorentz-Minkowski plane.

MSC: 53C50, 53C40

Keywords: Sturmian spirals, Lorentz-Minkowski plane, spacelike curve, timelike curve

\section{Contents}

1 Introduction $\quad 25$

2 Preliminaries $\quad 26$

3 Spacelike Spirals in Lorentz-Minkowski Plane 27

3.1 Spacelike Sturmian Spirals . . . . . . . . . . . . . . . . . . 28

3.2 Generalized Spacelike Sturmian Spirals . . . . . . . . . . . . . . . . . . 30

4 Timelike Sturmian Spirals in Lorentz-Minkowski Plane 34

4.1 Timelike Sturmian Spirals . . . . . . . . . . . . . . . . . . 35

4.2 Generalized Timelike Sturmian Spirals . . . . . . . . . . . . . . . 36

5 Conclusion $\quad 41$

References $\quad 41$

\section{Introduction}

The general theory of curves in Euclidean space (more generally in a Riemannian manifold) has been developed long time ago and we have a deep knowledge of their local and global geometries [7]. When the ambient space is a Lorentz-Minkowski 
space (and more generally a semi-Riemannian space or a semi-Riemannian manifold), our knowledge is definitely more restricted in comparison with the Euclidean case.

Nevertheless the fundamental existence and uniqueness theorem in the theory of plane curves which states that a curve is uniquely determined (up to Euclidean motion) by its curvature given as a function of its arc-length (see [1] and [12]) is still valid. However, again the simplicity of the situation is elusive as in many cases it is impossible to find the curve explicitly.

One should notice also that while some classes of space curves in the LorentzMinkowski space like helices, Bertrand and Mannheim curves (see [3-5], [8]), are well documented as in the Euclidean case, the studies on planar curves in pseudoEuclidean plane are not so many. For example, up to our knowledge, spiral curves are not studied in the Lorentz-Minkowski plane. They are representatives of the plane curves whose curvature depends solely on the distance from the origin in the Euclidean plane. These class of curves was already studied in some detail in $[2,13]$ and [14] where it was proven that they could be reconstructed via quadratures.

Also, Singer [13] proved that the problem of determining a curve whose curvature is $\kappa(r)$, where $r$ is the distance from the origin, is solvable by quadratures. It is known that Sturmian spirals provide examples of such curves. These curves and their generalizations were studied in [10]. Here we take the same problem in Lorentz-Minkowski plane and consider both spacelike and timelike plane curves. The paper is organized as follows: Section 2 reviews some basic notions in Lorentz-Minkowski plane. In Section 3, we consider the spacelike Sturmian spirals and their generalization. Section 4 deals with their timelike analogues. All cases are presented by analytic formulas and illustrated graphically.

\section{Preliminaries}

The Lorentz-Minkowski plane $\mathbb{E}_{1}^{2}$ is the Euclidean plane $\mathbb{E}^{2}$ equipped with indefinite flat metric given by

$$
g=-\mathrm{d} x^{2}+\mathrm{d} z^{2}
$$

where $(x, z)$ are the rectangular coordinates on $\mathbb{E}_{1}^{2}$. Recall that a vector $v \in$ $\mathbb{E}_{1}^{2} \backslash\{0\}$ is spacelike if $g(v, v)>0$, timelike if $g(v, v)<0$ and null (lightlike) if $g(v, v)=0$. In particular, the vector $v=0$ is said to be a spacelike. The norm of a vector $v$ is given by $\|v\|=\sqrt{|g(v, v)|}$. Two vectors $v$ and $w$ are said to be orthogonal, if $g(v, w)=0$. An arbitrary curve $\alpha(s)$ in $\mathbb{E}_{1}^{2}$, can locally be spacelike, timelike, if all its velocity vectors $\alpha^{\prime}(s)$ are respectively spacelike, timelike. 
A spacelike or timelike curve $\alpha$ is parametrized by the arclength parameter $s$ if $g\left(\alpha^{\prime}(s), \alpha^{\prime}(s)\right)= \pm 1([11])$.

A spacelike curve in Lorentz-Minkowski plane can be parametrized ([6], [7], [9]) as

$$
\mathbf{x}(s)=\left(\int_{0}^{s} \sinh \Phi(s) \mathrm{d} s, \int_{0}^{s} \cosh \Phi(s) \mathrm{d} s\right)
$$

with the Frenet vector fields

$$
\mathbf{T}(s)=(\sinh \Phi(s), \cosh \Phi(s)), \quad \mathbf{N}(s)=(\cosh \Phi(s), \sinh \Phi(s))
$$

and the curvature function

$$
\kappa(s)=g\left(\frac{\mathrm{d} \mathbf{T}(s)}{\mathrm{d} s}, \mathbf{N}(s)\right)=-\frac{\mathrm{d} \Phi(s)}{\mathrm{d} s} .
$$

The Frenet vectors fields satisfy the relations

$$
\frac{\mathrm{d} \mathbf{x}}{\mathrm{d} s}=\mathbf{T}, \quad \frac{\mathrm{d} \mathbf{T}}{\mathrm{d} s}=-\kappa \mathbf{N}, \quad \frac{\mathrm{d} \mathbf{N}}{\mathrm{d} s}=-\kappa \mathbf{T} .
$$

A timelike curve in Lorentz-Minkowski plane can be parametrized [6] respectively as

$$
\mathbf{x}(s)=\left(\int_{0}^{s} \cosh \Phi(s) \mathrm{d} s, \int_{0}^{s} \sinh \Phi(s) \mathrm{d} s\right)
$$

with the Frenet vector fields

$$
\mathbf{T}(s)=(\cosh \Phi(s), \sinh \Phi(s)), \quad \mathbf{N}(s)=(\sinh \Phi(s), \cosh \Phi(s))
$$

and the curvature function

$$
\kappa(s)=g\left(\frac{\mathrm{d} \mathbf{T}(s)}{\mathrm{d} s}, \mathbf{N}(s)\right)=\frac{\mathrm{d} \Phi(s)}{\mathrm{d} s} .
$$

In this case the Frenet vectors fields satisfy the relations

$$
\frac{\mathrm{d} \mathbf{x}}{\mathrm{d} s}=\mathbf{T}, \quad \frac{\mathrm{d} \mathbf{T}}{\mathrm{d} s}=\kappa \mathbf{N}, \quad \frac{\mathrm{d} \mathbf{N}}{\mathrm{d} s}=\kappa \mathbf{T} .
$$

\section{Spacelike Spirals in Lorentz-Minkowski Plane}

In this section, we consider the spacelike Sturmian spirals and the generalized spacelike Sturmian spirals by using the following relation

$$
\kappa(s)=\frac{\sigma}{r}, \quad r=\sqrt{|g(\mathbf{x}(s), \mathbf{x}(s))|} .
$$


Let $\mathbf{x}$ is a spacelike curve and the position vector $\mathbf{x}$ be specified as

$$
\mathbf{x}(s)=\xi(s) \mathbf{T}(s)+\eta(s) \mathbf{N}(s)
$$

where $\xi$ and $\eta$ are differentiable functions of $s$. Differentiating (10) with respect to $s$ and using (4), we get

$$
\mathbf{T}=\left(\xi^{\prime}-\eta \kappa\right) \mathbf{T}+\left(\eta^{\prime}-\xi \kappa\right) \mathbf{N} .
$$

From (10) and (11), we have

$$
\xi^{\prime}=1+\eta \kappa, \quad \eta^{\prime}=\xi \kappa, \quad \kappa=\frac{\sigma}{r}=\frac{\sigma}{\sqrt{\varepsilon\left(\xi^{2}-\eta^{2}\right)}}
$$

where $\varepsilon= \pm 1$ is chosen so $\varepsilon\left(\xi^{2}-\eta^{2}\right) \geq 0$.

By multiplying the first equation in (12) by $\xi$, the second one by $\eta$ and summing the so obtained expressions, we find

$$
\xi=\varepsilon r r^{\prime} .
$$

Substituting back (13) into the second equation in (12) and integrating the so obtained expression we end up with the formula

$$
\eta=\varepsilon \int r \kappa(r) \mathrm{d} r+c
$$

where $c$ is the integration constant.

\subsection{Spacelike Sturmian Spirals}

By the very definition [15] of these curve, they have the property that at each point their curvature radius $\mathfrak{R}$ is equal to the distance $r$ from the origin. Thus, $\kappa$ is given by the third equation in (12) and $\sigma=1$.

From (13) and (14), we get

$$
\eta=\varepsilon r+c, \quad r^{\prime}=\frac{\varepsilon \sqrt{(1+\varepsilon) r^{2}+2 \varepsilon c r+c^{2}}}{r} .
$$

Now, we consider $\varepsilon=1$ and $\varepsilon=-1$ cases separately.

i) Let $\varepsilon=1$. Then we have

$$
\eta=r+c, \quad r^{\prime}=\frac{\sqrt{2 r^{2}+2 c r+c^{2}}}{r}, \quad c>0 .
$$


It is convenient to perform the respective integration in (16) by switching to a new independent variable $t$ defined by

$$
\frac{\mathrm{d} s}{\mathrm{~d} t}=r
$$

This leads to the following results

$$
\begin{array}{ll}
\xi(t)=\frac{c}{\sqrt{2}} \cosh (\sqrt{2} t), \quad \eta(t)=\frac{c}{2}(\sinh (\sqrt{2} t)+1) \\
r(t)=\frac{c}{2}(\sinh (\sqrt{2} t)-1), \quad \Phi=-t .
\end{array}
$$

By rewriting (10) in its components, we obtain the relations

$$
x=\xi \sinh \Phi+\eta \cosh \Phi, \quad z=\xi \cosh \Phi+\eta \sinh \Phi
$$

which combined with the above findings provide the sought parameterization of the Sturmian spirals

$$
\begin{aligned}
& x=-\frac{c}{\sqrt{2}} \cosh (\sqrt{2} t) \sinh t+\frac{c}{2}(\sinh (\sqrt{2} t)+1) \cosh t \\
& z=\frac{c}{\sqrt{2}} \cosh (\sqrt{2} t) \cosh t-\frac{c}{2}(\sinh (\sqrt{2} t)+1) \sinh t
\end{aligned}
$$

ii) Let $\varepsilon=1$ and $c=0$. Then we have

$$
\begin{aligned}
& \xi(s)=2 s+\sqrt{2} d, \quad \eta(s)=\sqrt{2} s+d, \quad d \in \mathbb{R} \\
& r(s)=\sqrt{2} s+d, \quad \Phi(s)=-\frac{1}{\sqrt{2}} \ln |\sqrt{2} s+d| .
\end{aligned}
$$

Thus, it can be easily obtained that

$$
\begin{aligned}
& x=(2 s+\sqrt{2} d) \sinh \Phi+(\sqrt{2} s+d) \cosh \Phi \\
& z=(2 s+\sqrt{2} d) \cosh \Phi+(\sqrt{2} s+d) \sinh \Phi .
\end{aligned}
$$

iii) Let $\varepsilon=-1$. Then from (15) we get

$$
\eta=-r+c, \quad r^{\prime}=\frac{-\sqrt{c^{2}-2 c r}}{r}, \quad c<0 .
$$

Exchanging as before the arc-length parameter with $t$ via (17), we obtain

$$
\xi(t)=c t, \quad \eta(t)=\frac{c}{2}\left(1+t^{2}\right), \quad r(t)=\frac{c}{2}\left(1-t^{2}\right)
$$


where $t \in(-\infty,-1) \cup(1, \infty)$.

The integration of (3) leads to

$$
\Phi=-t
$$

which combined with (19) produces the parametrization

$$
\begin{aligned}
& x=-c t \sinh t+\frac{c}{2}\left(1+t^{2}\right) \cosh t \\
& z=c t \cosh t-\frac{c}{2}\left(1+t^{2}\right) \sinh t .
\end{aligned}
$$
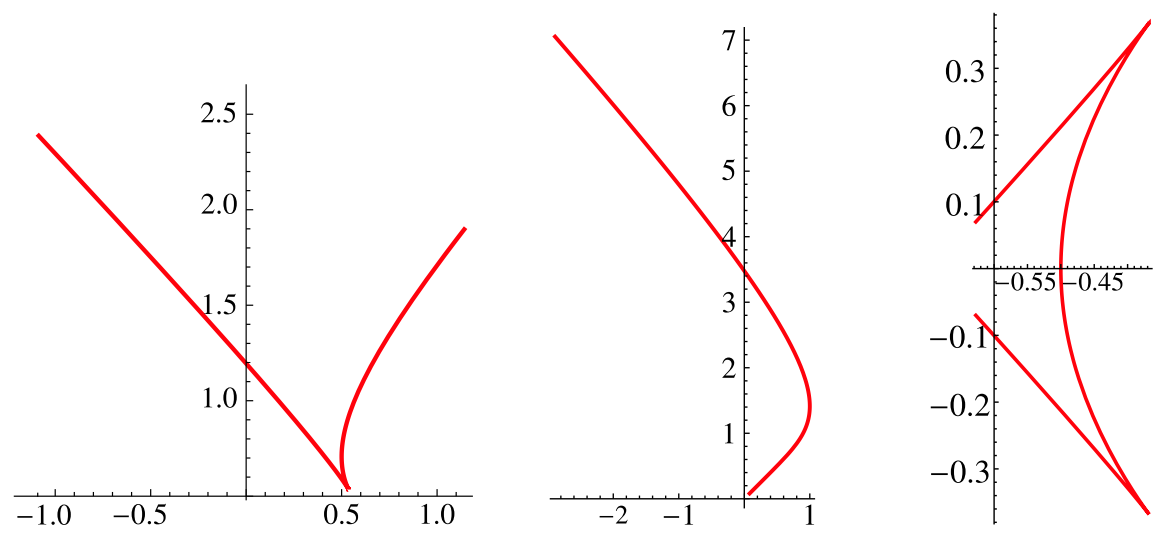

Figure 1. The standard spacelike Sturmian spirals generated by (20) with $c=1$ (left), by (22) with $d=0$ (middle) and by (26) with $c=-1$ (right).

\subsection{Generalized Spacelike Sturmian Spirals}

Forgetting about the restriction on the value of $\sigma$ in previous section, one can consider as well the other two obvious possibilities, $\sigma>1$ and $0<\sigma<1$ which have to be viewed as a generalization of the spacelike Sturmian spirals.

Case 1. Let $\sigma>1$. Then from (13) and (14), we get

$$
\xi=\sqrt{\sigma_{\varepsilon}^{2} r^{2}+2 \varepsilon \sigma c r+c^{2}}, \quad \eta=\varepsilon \sigma r+c, \quad \sigma_{\varepsilon}=\sqrt{\sigma^{2}+\varepsilon}
$$

and

$$
r^{\prime}=\frac{\varepsilon \sqrt{\sigma_{\varepsilon}^{2} r^{2}+2 \varepsilon \sigma c r+c^{2}}}{r} .
$$

Now, let us consider again the cases $\varepsilon=1$ and $\varepsilon=-1$ separately. 
i) Let $\varepsilon=1$. Then we have

$$
\eta=\sigma r+c, \quad c \in \mathbb{R}
$$

and

$$
r^{\prime}=\frac{\sqrt{\sigma_{+}^{2} r^{2}+2 \sigma c r+c^{2}}}{r}, \quad \sigma_{+}=\sqrt{\sigma^{2}+1} .
$$

Changing the parameter as in (17), we get

$$
\begin{aligned}
\xi(t) & =\frac{c}{\sigma_{+}} \cosh \left(\sigma_{+} t\right), & \eta(t) & =\frac{c}{\sigma_{+}^{2}}\left(\sigma \sinh \left(\sigma_{+} t\right)+1\right) \\
r(t) & =\frac{c}{\sigma_{+}^{2}}\left(\sinh \left(\sigma_{+} t\right)-\sigma\right), & \Phi & =-\sigma t .
\end{aligned}
$$

Further on, by making use of formula (10) one finds easily the parametrization of the generalized Sturmian spirals

$$
\begin{aligned}
& x=\left[-\frac{c}{\sigma_{+}} \cosh \left(\sigma_{+} t\right)\right] \sinh (\sigma t)+\left[\frac{c}{\sigma_{+}^{2}}\left(\sigma \sinh \left(\sigma_{+} t\right)+1\right)\right] \cosh (\sigma t) \\
& z=\left[\frac{c}{\sigma_{+}} \cosh \left(\sigma_{+} t\right)\right] \cosh (\sigma t)-\left[\frac{c}{\sigma_{+}^{2}}\left(\sigma \sinh \left(\sigma_{+} t\right)+1\right)\right] \sinh (\sigma t) .
\end{aligned}
$$

ii) Let $\varepsilon=-1$. Then from (27) and (28), we get

$$
\xi=-\sqrt{\sigma_{-}^{2} r^{2}-2 \sigma c r+c^{2}}, \quad \eta=-\sigma r+c, \quad \sigma_{-}=\sqrt{\sigma^{2}-1}, \quad c>0
$$

and

$$
r^{\prime}=-\frac{\sqrt{\sigma_{-}^{2} r^{2}-2 \sigma c r+c^{2}}}{r} .
$$

One easily concludes that the expression under the radical on the right-hand side is positive provided that $c>0$ and if $r$ lies in the interval

$$
\frac{c}{\sigma+1}<r<\frac{c}{\sigma-1}
$$

Passing to the $t$-parametrization (17), we find

$$
\begin{array}{ll}
\xi(t)=-\frac{c}{\sigma_{-}} \sinh \left(\sigma_{-} t\right), \quad \eta(t)=-\frac{c}{\sigma_{-}^{2}}\left(\sigma \cosh \left(\sigma_{-} t\right)+1\right) \\
r(t)=\frac{c}{\sigma_{-}^{2}}\left(\cosh \left(\sigma_{-} t\right)+\sigma\right), \quad \Phi=-\sigma t .
\end{array}
$$


By using (36) in (10), we get the components of the Sturmian spirals

$$
\begin{aligned}
& x=\left[\frac{c}{\sigma_{-}} \sinh \left(\sigma_{-} t\right)\right] \sinh (\sigma t)-\left[\frac{c}{\sigma_{-}^{2}}\left(\sigma \cosh \left(\sigma_{-} t\right)+1\right)\right] \cosh (\sigma t) \\
& z=\left[-\frac{c}{\sigma_{-}} \sinh \left(\sigma_{-} t\right)\right] \cosh (\sigma t)+\left[\frac{c}{\sigma_{-}^{2}}\left(\sigma \cosh \left(\sigma_{-} t\right)+1\right)\right] \sinh (\sigma t) .
\end{aligned}
$$

Case 2. Let $\sigma>1$ and $c=0$. Then from (27) and (28), we obtain

$$
\xi=\sigma_{\varepsilon} r, \quad \eta=\varepsilon \sigma r, \quad r^{\prime}=\varepsilon \sigma_{\varepsilon} .
$$

These lead further to the useful formulas

$$
\begin{aligned}
& \xi(s)=\varepsilon \sigma_{\varepsilon}^{2} s+d \sigma_{\varepsilon}, \quad \eta(s)=\sigma \sigma_{\varepsilon} s+d \varepsilon \sigma, \quad d \in \mathbb{R} \\
& r(s)=\varepsilon \sigma_{\varepsilon} s+d, \quad \Phi(s)=-\frac{\varepsilon \sigma}{\sigma_{\varepsilon}} \ln \left|\varepsilon \sigma_{\varepsilon} s+d\right| .
\end{aligned}
$$

Inserting these results in (10) one finds the parametrization

$$
\begin{aligned}
& x=\left[\varepsilon \sigma_{\varepsilon}^{2} s+d \sigma_{\varepsilon}\right] \sinh \Phi+\left[\sigma \sigma_{\varepsilon} s+d \varepsilon \sigma\right] \cosh \Phi \\
& z=\left[\varepsilon \sigma_{\varepsilon}^{2} s+d \sigma_{\varepsilon}\right] \cosh \Phi+\left[\sigma \sigma_{\varepsilon} s+d \varepsilon \sigma\right] \sinh \Phi .
\end{aligned}
$$
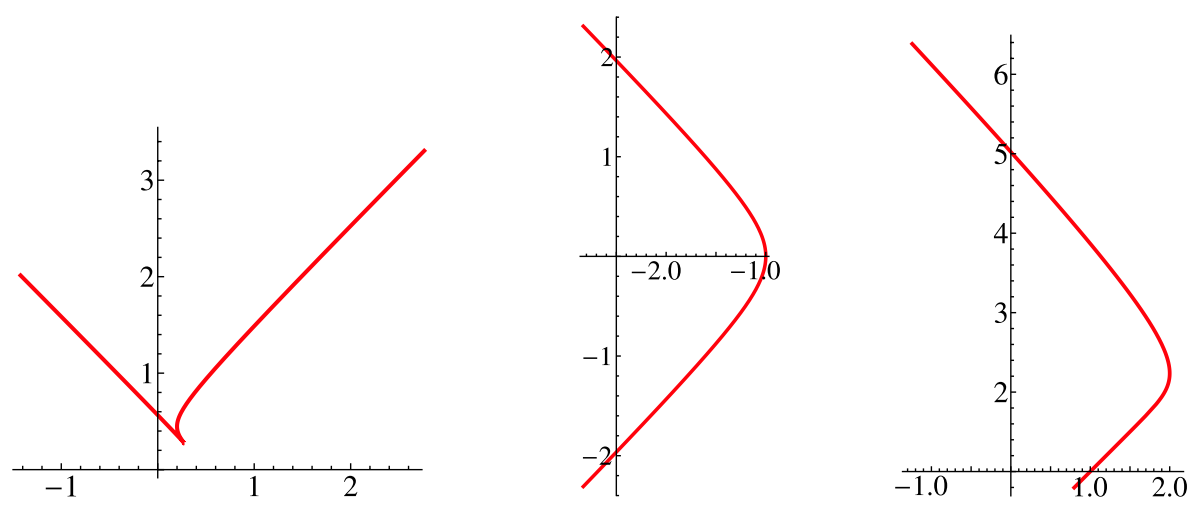

Figure 2. The generalized spacelike Sturmian spirals generated by (32) with $c=1$ and $\sigma=2$ (left), by (37) with $c=1$ and $\sigma=2$ (middle), by (40) with $d=0$ and $\varepsilon=1$ (right).

Case 3. Let $0<\sigma<1$. This time via (27) and (28), we get

$$
\xi=\sqrt{\sigma_{\varepsilon}^{2} r^{2}+2 \varepsilon \sigma c r+c^{2}}, \quad \eta=\varepsilon \sigma r+c, \quad c \in \mathbb{R}
$$


and

$$
r^{\prime}=\frac{\varepsilon \sqrt{\sigma_{\varepsilon}^{2} r^{2}+2 \varepsilon \sigma c r+c^{2}}}{r} .
$$

As before we consider $\varepsilon=1$ and $\varepsilon=-1$ cases separately.

i) Let $\varepsilon=1$. Then we have

$$
\eta=\sigma r+c, \quad r^{\prime}=\frac{\sqrt{\sigma_{+}^{2} r^{2}+2 \sigma c r+c^{2}}}{r} .
$$

By going to the new independent variable $t$ as in (17), we find

$$
\begin{aligned}
& \xi(t)=\frac{c}{\sigma_{+}} \cosh \left(\sigma_{+} t\right), \quad \eta(t)=\frac{c}{\sigma_{+}^{2}}\left(\sigma \sinh \left(\sigma_{+} t\right)+1\right) \\
& r(t)=\frac{c}{\sigma_{+}^{2}}\left(\sinh \left(\sigma_{+} t\right)-\sigma\right), \quad \Phi=-\sigma t
\end{aligned}
$$

Rewriting (10) in its components, we obtain the parametrization

$$
\begin{aligned}
& x=\left[-\frac{c}{\sigma_{+}} \cosh \left(\sigma_{+} t\right)\right] \sinh (\sigma t)+\left[\frac{c}{\sigma_{+}^{2}}\left(\sigma \sinh \left(\sigma_{+} t\right)+1\right)\right] \cosh (\sigma t) \\
& z=\left[\frac{c}{\sigma_{+}} \cosh \left(\sigma_{+} t\right)\right] \cosh (\sigma t)-\left[\frac{c}{\sigma_{+}^{2}}\left(\sigma \sinh \left(\sigma_{+} t\right)+1\right)\right] \sinh (\sigma t) .
\end{aligned}
$$

ii) Let $\varepsilon=-1$. Then from (27) and (28), we get

$$
\eta=-\sigma r+c, \quad r^{\prime}=-\frac{\sqrt{\sigma_{-}^{2} r^{2}-2 \sigma c r+c^{2}}}{r} .
$$

One easily concludes that the expression under the radical on the right-hand side is positive provided that

$$
c>0 \text { and } 0<r<\frac{c}{\sigma+1}
$$

By the similar techniques used till now we obtain

$$
\begin{aligned}
& \xi(t)=\frac{c}{\omega} \cos (\omega t), \quad \eta(t)=\frac{c}{\omega^{2}}(1+\sigma \sin (\omega t)), \quad \omega=\sqrt{1-\sigma^{2}} \\
& r(t)=\frac{c}{\omega^{2}}(-\sin (\omega t)+\sigma), \quad t \in\left[-\frac{\pi}{2 \omega}, \frac{\pi}{2 \omega}\right], \quad \Phi=-\sigma t .
\end{aligned}
$$


Substituting (44) in (10), we have

$$
\begin{aligned}
& x=\left[-\frac{c}{\omega} \cos (\omega t)\right] \sinh (\sigma t)+\left[\frac{c}{\omega^{2}}(1+\sigma \sin (\omega t))\right] \cosh (\sigma t) \\
& z=\left[\frac{c}{\omega} \cos (\omega t)\right] \cosh (\sigma t)-\left[\frac{c}{\omega^{2}}(1+\sigma \sin (\omega t))\right] \sinh (\sigma t) .
\end{aligned}
$$

Case 4. Let $0<\sigma<1, c=0$ and $\varepsilon=1$. Then we have

$$
\begin{aligned}
& \xi(s)=\sigma_{+}^{2} s+d \sigma_{+}, \quad \eta(s)=\sigma \sigma_{+} s+\sigma d \\
& r(s)=\sigma_{+} s+d, \quad \Phi(s)=-\frac{\sigma}{\sigma_{+}} \ln \left|\sigma_{+} s+d\right| .
\end{aligned}
$$

Considering the equations (10) and (48) together, we find the following

$$
\begin{aligned}
& x=\left[\sigma_{+}^{2} s+d \sigma_{+}\right] \sinh \Phi+\left[\sigma \sigma_{+} s+\sigma d\right] \cosh \Phi \\
& z=\left[\sigma_{+}^{2} s+d \sigma_{+}\right] \cosh \Phi+\left[\sigma \sigma_{+} s+\sigma d\right] \sinh \Phi .
\end{aligned}
$$
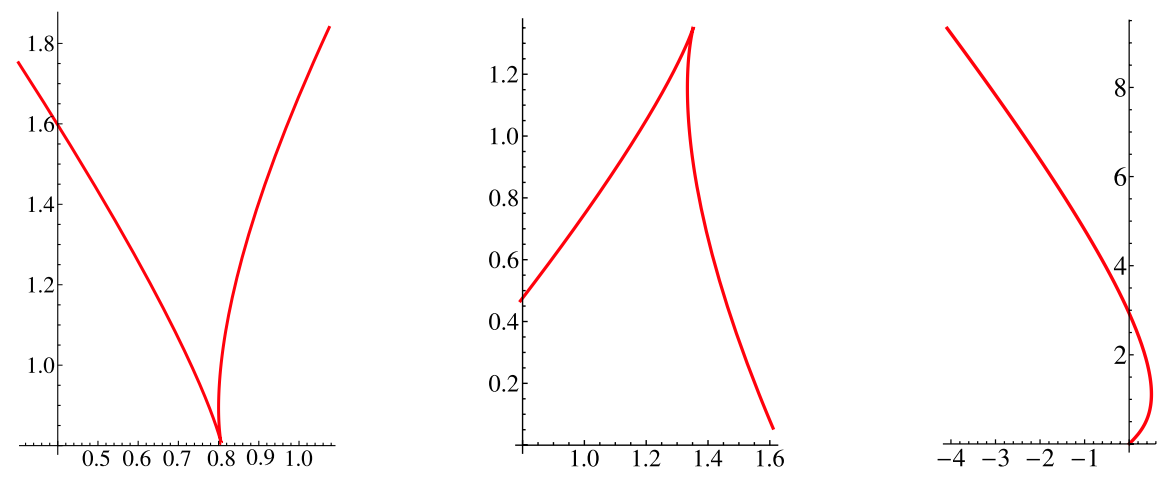

Figure 3. The generalized spacelike Sturmian spirals generated by (45) with $c=1, \sigma=\frac{1}{2}$ (left) by (49) with $c=1, \sigma=\frac{1}{2}$ (middle) and by (50) with $d=\frac{1}{2}$ and $\sigma=\frac{1}{2}$ (right).

\section{Timelike Sturmian Spirals in Lorentz-Minkowski Plane}

In this section, we study the timelike Sturm spirals and the generalized timelike Sturmian spirals by considering relations (9).

Let $\mathbf{x}$ be a timelike curve and the position vector $\mathrm{x}$ is presented in the form

$$
\mathbf{x}(s)=\xi(s) \mathbf{T}(s)+\eta(s) \mathbf{N}(s)
$$


where $\xi$ and $\eta$ are differentiable functions of $s$.

Differentiating (51) with respect to $s$ and using (8), we get

$$
\mathbf{T}=\left(\xi^{\prime}+\eta \kappa\right) \mathbf{T}+\left(\eta^{\prime}+\xi \kappa\right) \mathbf{N} .
$$

From (51) and (52), we have

$$
\xi^{\prime}=1-\eta \kappa, \quad \eta^{\prime}=-\xi \kappa, \quad \kappa=\frac{\sigma}{r}=\frac{\sigma}{\sqrt{\varepsilon\left(\eta^{2}-\xi^{2}\right)}}
$$

where $\varepsilon= \pm 1$ and such that $\varepsilon\left(\eta^{2}-\xi^{2}\right) \geq 0$.

Multiplying the first equation above by $\xi$, the second one by $\eta$ and summing the so obtained expressions we end up with the equation

$$
\xi=-\varepsilon r r^{\prime}
$$

Substituting back the relation (54) into the second equation of (53) and integrating we have finally

$$
\eta=\varepsilon \int r \kappa(r) \mathrm{d} r+c
$$

where $c$ is the integration constant.

\subsection{Timelike Sturmian Spirals}

From [15] these curves have the property that at each point their curvature radius $\mathfrak{R}$ is equal to the distance $r$ from the origin. Thus, $\kappa$ is given by the third formula in (53) in which $\sigma=1$. From (54) and (55) we have

$$
\eta=\varepsilon r+c, \quad r^{\prime}=\frac{-\varepsilon \sqrt{(1-\varepsilon) r^{2}+2 \varepsilon c r+c^{2}}}{r}, \quad c>0
$$

and let us consider again the cases $\varepsilon=1$ and $\varepsilon=-1$ separately.

i) Let $\varepsilon=1$. Then we have

$$
\eta=r+c, \quad r^{\prime}=\frac{-\sqrt{2 c r+c^{2}}}{r} .
$$

Using (17), we get the followings

$$
\xi(t)=c t, \quad \eta(t)=\frac{c}{2}\left(t^{2}+1\right), \quad r(t)=\frac{c}{2}\left(t^{2}-1\right), \quad \Phi=t .
$$

By rewriting (51) in its components, we obtain the relations

$$
x=\xi \cosh \Phi+\eta \sinh \Phi, \quad z=\xi \sinh \Phi+\eta \cosh \Phi
$$


which combined with the above findings leads to the parametrization of the Sturm spirals

$$
x=c t \cosh t+\frac{c}{2}\left(t^{2}+1\right) \sinh t, \quad z=c t \sinh t+\frac{c}{2}\left(t^{2}+1\right) \cosh t .
$$

ii) Let $\varepsilon=-1$. Then from (56) we have directly

$$
\eta=-r+c, \quad r^{\prime}=\frac{\sqrt{2 r^{2}-2 c r+c^{2}}}{r}, \quad c>0 .
$$

Changing the parameter like in (17), leads to

$$
\begin{aligned}
& \xi(t)=\frac{c}{\sqrt{2}} \cosh (\sqrt{2} t), \quad \eta(t)=\frac{c}{2}(1-\sinh (\sqrt{2} t)) \\
& r(t)=\frac{c}{2}(1+\sinh (\sqrt{2} t)), \quad \Phi=t .
\end{aligned}
$$

By using (51), we get finally

$$
\begin{aligned}
& x=\frac{c}{\sqrt{2}} \cosh (\sqrt{2} t) \cosh t+\frac{c}{2}(1-\sinh (\sqrt{2} t)) \sinh t \\
& z=\frac{c}{\sqrt{2}} \cosh (\sqrt{2} t) \sinh t+\frac{c}{2}(1-\sinh (\sqrt{2} t)) \cosh t .
\end{aligned}
$$

iii) Let $\varepsilon=-1$ and $c=0$. Then we have

$$
\begin{aligned}
& \xi(s)=2 s+\sqrt{2} d, \quad \eta(s)=-\sqrt{2} s-d, \quad d \in \mathbb{R} \\
& r(s)=\sqrt{2} s+d, \quad \Phi(s)=\frac{1}{\sqrt{2}} \ln |\sqrt{2} s+d| .
\end{aligned}
$$

Thus, it can be easily obtained that

$$
\begin{aligned}
& x=(2 s+\sqrt{2} d) \cosh \Phi+(-\sqrt{2} s-d) \sinh \Phi \\
& z=(2 s+\sqrt{2} d) \sinh \Phi+(-\sqrt{2} s-d) \cosh \Phi .
\end{aligned}
$$

\subsection{Generalized Timelike Sturmian Spirals}

Disregarding the restriction on the value of $\sigma$, one can consider as well the other two obvious possibilities, $\sigma>1$ and $0<\sigma<1$ which have to be viewed as generalizations of the timelike Sturmian spirals. 

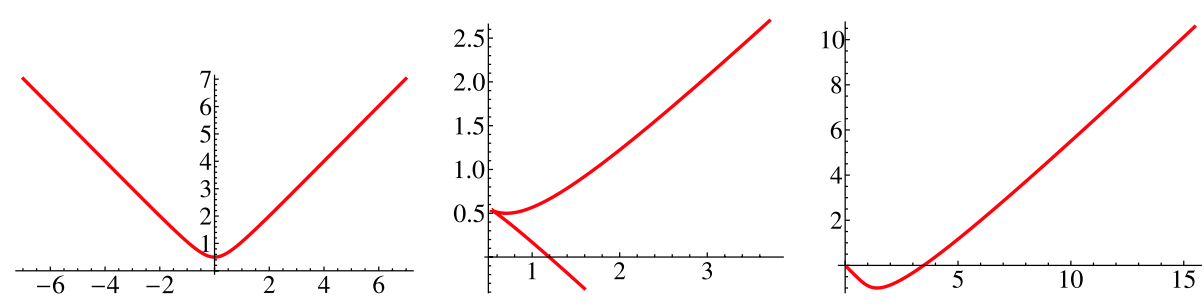

Figure 4. The standard timelike Sturmian spirals generated by $(60)$ with $c=1$ (left), (64) with $c=1$ (middle) and (66) with $d=0$ (right).

Case 5. Let $\sigma>1$. Then from (54) and (55), we get

$$
\xi=\sqrt{\left(\sigma^{2}-\varepsilon\right) r^{2}+2 \varepsilon \sigma c r+c^{2}}, \quad \eta=\varepsilon \sigma r+c
$$

and

$$
r^{\prime}=-\frac{\varepsilon \sqrt{\sigma_{\varepsilon}^{2} r^{2}+2 \varepsilon \sigma c r+c^{2}}}{r} .
$$

Let us consider the cases $\varepsilon=1$ and $\varepsilon=-1$ separately.

i) Let $\varepsilon=1$. Then we have

$$
\eta=\sigma r+c, \quad r^{\prime}=-\frac{\sqrt{\sigma_{-}^{2} r^{2}+2 \sigma c r+c^{2}}}{r}, \quad c>0 .
$$

Using as independent variable $t$ and (51), we find

$$
\begin{array}{ll}
\xi(t)=-\frac{c}{\sigma_{-}} \sinh \left(\sigma_{-} t\right), \quad \eta(t)=\frac{c}{\sigma_{-}^{2}}\left(\sigma \cosh \left(\sigma_{-} t\right)-1\right) \\
r(t)=\frac{c}{\sigma_{-}^{2}}\left(\cosh \left(\sigma_{-} t\right)-\sigma\right), \quad \Phi=\sigma t .
\end{array}
$$

If we rewrite (51) in its components, we get the parametrization

$$
\begin{aligned}
& x=\left[-\frac{c}{\sigma_{-}} \sinh \left(\sigma_{-} t\right)\right] \cosh \sigma t+\left[\frac{c}{\sigma_{-}^{2}}\left(\sigma \cosh \left(\sigma_{-} t\right)-1\right)\right] \sinh \sigma t \\
& z=\left[-\frac{c}{\sigma_{-}} \sinh \left(\sigma_{-} t\right)\right] \sinh \sigma t+\left[\frac{c}{\sigma_{-}^{2}}\left(\sigma \cosh \left(\sigma_{-} t\right)-1\right)\right] \cosh \sigma t .
\end{aligned}
$$

ii) Let $\varepsilon=-1$. Then from (67) and (68), we get

$$
\xi=\sqrt{\sigma_{+}^{2} r^{2}-2 \sigma c r+c^{2}}, \quad \eta=-\sigma r+c, \quad c>0
$$


and

$$
r^{\prime}=\frac{\sqrt{\sigma_{+}^{2} r^{2}-2 \sigma c r+c^{2}}}{r} .
$$

By a similar change of the parameter as in (17), we have

$$
\begin{aligned}
& \xi(t)=\frac{c}{\sigma_{+}} \cosh \left(\sigma_{+} t\right), \quad \eta(t)=\frac{c}{\sigma_{+}^{2}}\left(1-\sigma \sinh \left(\sigma_{+} t\right)\right) \\
& r(t)=\frac{c}{\sigma_{+}^{2}}\left(\sinh \left(\sigma_{+} t\right)+\sigma\right), \quad \Phi=\sigma t .
\end{aligned}
$$

Relying on the equations (51) and (74) one gets at the end

$$
\begin{aligned}
& x=\left[\frac{c}{\sigma_{+}} \cosh \left(\sigma_{+} t\right)\right] \cosh \sigma t+\left[\frac{c}{\sigma_{+}^{2}}\left(1-\sigma \sinh \left(\sigma_{+} t\right)\right)\right] \sinh \sigma t \\
& z=\left[\frac{c}{\sigma_{+}} \cosh \left(\sigma_{+} t\right)\right] \sinh \sigma t+\left[\frac{c}{\sigma_{+}^{2}}\left(1-\sigma \sinh \left(\sigma_{+} t\right)\right)\right] \cosh \sigma t
\end{aligned}
$$

Case 6. Let $\sigma>1$ and $c=0$. Then from (67) and (68), we obtain

$$
\xi=\sqrt{\sigma^{2}-\varepsilon}, \quad \eta=\varepsilon \sigma r, \quad r^{\prime}=-\varepsilon \sqrt{\sigma^{2}-\varepsilon} .
$$

It can be easily obtained that

$$
\begin{aligned}
& \xi(s)=-\varepsilon\left(\sigma^{2}-\varepsilon\right) s+d \sqrt{\sigma^{2}-\varepsilon}, \quad \eta(s)=-\sigma \sqrt{\sigma^{2}-\varepsilon} s+d \varepsilon \sigma \\
& r(s)=-\varepsilon \sqrt{\sigma^{2}-\varepsilon} s+d, \quad \Phi(s)=\frac{-\varepsilon \sigma}{\sqrt{\sigma^{2}-\varepsilon}} \ln \left|-\varepsilon \sqrt{\sigma^{2}-\varepsilon} s+d\right| .
\end{aligned}
$$

Thus, we have

$$
\begin{aligned}
& x=\left(-\varepsilon\left(\sigma^{2}-\varepsilon\right) s+d \sqrt{\sigma^{2}-\varepsilon}\right) \cosh \Phi+\left(-\sigma \sqrt{\sigma^{2}-\varepsilon} s+d \varepsilon \sigma\right) \sinh \Phi \\
& z=\left(-\varepsilon\left(\sigma^{2}-\varepsilon\right) s+d \sqrt{\sigma^{2}-\varepsilon}\right) \sinh \Phi+\left(-\sigma \sqrt{\sigma^{2}-\varepsilon} s+d \varepsilon \sigma\right) \cosh \Phi .
\end{aligned}
$$

Case 7. Let $0<\sigma<1$. Then via (67) and (68), we get

$$
\xi=\sqrt{\left(\sigma^{2}-\varepsilon\right) r^{2}+2 \varepsilon \sigma c r+c^{2}}, \quad \eta=\varepsilon \sigma r+c
$$

and

$$
r^{\prime}=\frac{-\varepsilon \sqrt{\left(\sigma^{2}-\varepsilon\right) r^{2}+2 \varepsilon \sigma c r+c^{2}}}{r}
$$



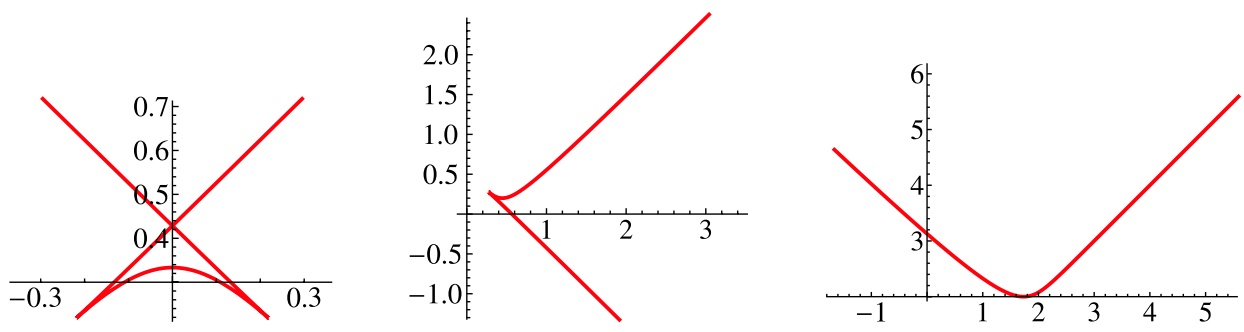

Figure 5. The generalized timelike Sturmian spirals generated by (71) with $c=1, \sigma=2$ (left), by (75) with $c=1, \sigma=2$ (middle), and via (77) with $d=0, \varepsilon=1$ and $\sigma=2$ (right).

Now, we consider $\varepsilon=1$ or $\varepsilon=-1$ separately.

i) Let $\varepsilon=1$. Then we have

$$
\eta=\sigma r+c, \quad r^{\prime}=\frac{-\sqrt{\left(\sigma^{2}-1\right) r^{2}+2 \sigma c r+c^{2}}}{r} .
$$

One easily concludes that the expression under the radical on the right-hand side is positive provided that

$$
c>0 \quad \text { and } \quad 0<r<-\frac{c}{\sigma+1} .
$$

Using new independent variable, we find

$$
\begin{aligned}
& \xi(t)=\frac{c}{\omega} \cosh (\omega t), \quad \eta(t)=\frac{c}{\omega^{2}}(-\sigma \sinh (\omega t)+1) \\
& r(t)=\frac{c}{\omega^{2}}(-\sinh (\omega t)+\sigma), \quad \Phi=\sigma t
\end{aligned}
$$

This time equations (51) and (81) give rise to

$$
\begin{aligned}
& x=\left[\frac{c}{\omega} \cosh (\omega t)\right] \cosh \sigma t+\left[\frac{c}{\omega^{2}}(-\sigma \sinh (\omega t)+1)\right] \sinh \sigma t \\
& z=\left[\frac{c}{\omega} \cosh (\omega t)\right] \sinh \sigma t+\left[\frac{c}{\omega^{2}}(-\sigma \sinh (\omega t)+1)\right] \cosh \sigma t
\end{aligned}
$$

ii) Let $\varepsilon=-1$. Then from (67) and (68), we get

$$
\eta=-\sigma r+c, \quad r^{\prime}=\frac{\sqrt{\sigma_{+}^{2} r^{2}-2 \sigma c r+c^{2}}}{r} \quad c>0 .
$$


By using as independent variable $t$ defined in (17), we find immediately

$$
\begin{aligned}
& \xi(t)=\frac{c}{\sigma_{+}} \cos \left(\sigma_{+} t\right), \quad \eta(t)=\frac{c}{\sigma_{+}^{2}}\left(1-\sigma \sin \left(\sigma_{+} t\right)\right) \\
& r(t)=\frac{c}{\sigma_{+}^{2}}\left(\sin \left(\sigma_{+} t\right)+\sigma\right), \quad \Phi=\sigma t, \quad t \in\left[-\frac{\pi}{2 \sigma_{+}}, \frac{\pi}{2 \sigma_{+}}\right] .
\end{aligned}
$$

From (51) and (84), we reach the equations below

$$
\begin{aligned}
& x=\left[\frac{c}{\sigma_{+}} \cos \left(\sigma_{+} t\right)\right] \cosh \sigma t+\left[\frac{c}{\sigma_{+}^{2}}\left(1-\sigma \sin \left(\sigma_{+} t\right)\right)\right] \sinh \sigma t \\
& z=\left[\frac{c}{\sigma_{+}} \cos \left(\sigma_{+} t\right)\right] \sinh \sigma t+\left[\frac{c}{\sigma_{+}^{2}}\left(1-\sigma \sin \left(\sigma_{+} t\right)\right)\right] \cosh \sigma t .
\end{aligned}
$$
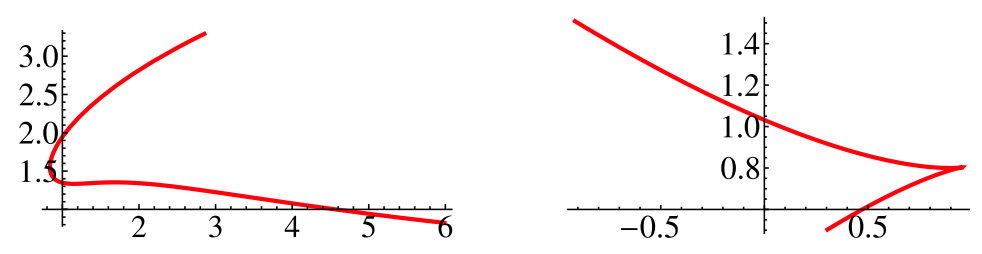

Figure 6. The generalized timelike Sturmian spirals generated by (82) with $c=1, \sigma=\frac{1}{2}$ (left), and (85) with $c=1, \sigma=\frac{1}{2}$ (right).

Case 8. Let $0<\sigma<1, c=0$ and $\varepsilon=-1$. Then we get

$$
\begin{array}{ll}
\xi(s)=\sigma_{+}^{2} s+d \sigma_{+}, & \eta(s)=-\sigma \sigma_{+} s-\sigma d \\
r(s)=\sigma_{+} s+d, & \Phi(s)=\frac{\sigma}{\sigma_{+}} \ln \left|\sigma_{+} s+d\right| .
\end{array}
$$

By rewriting (51) in its components, we get

$$
\begin{aligned}
& x=\left[\left(\sigma_{+}^{2}\right) s+d \sigma_{+}\right] \cosh \Phi+\left[-\sigma \sigma_{+} s-\sigma d\right] \sinh \Phi \\
& z=\left[\left(\sigma_{+}^{2}\right) s+d \sigma_{+}\right] \sinh \Phi+\left[-\sigma \sigma_{+} s-\sigma d\right] \cosh \Phi .
\end{aligned}
$$



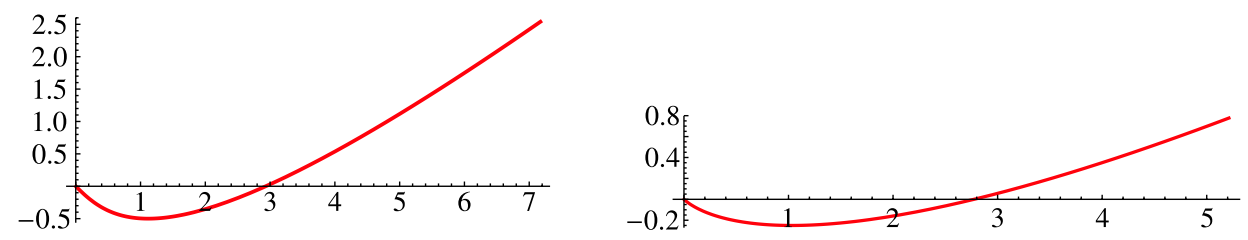

Figure 7. The generalized timelike Sturmian spirals generated by (86) with $d=2$ and $\sigma=\frac{1}{2}$ (left), with $d=0$ and $\sigma=\frac{1}{4}$ (right).

\section{Conclusion}

In [3], Mladenov et al have studied Sturmian spirals, planar curves whose curvature functions obey to the relation $\kappa(s)=\frac{\sigma}{r}$, where $r \in \mathbb{R}^{+}$is the distance from the origin in the Euclidean plane $\mathbb{E}^{2}$. By drawing inspiration from this work, here we consider spacelike and timelike Sturmian spirals in the Lorentz-Minkowski plane $\mathbb{E}_{1}^{2}$. More precisely we obtain the parametric equations of spacelike and timelike Sturmian spirals, generalized spacelike and timelike Sturmian spirals in the Lorentz-Minkowski plane. We present many figures of these curves and the graphics are realized using Mathematica. Further, we believe that the results in this paper suggest that other curves like Serret's curves, Bernoulli's Lemniscate and the plane curves whose curvatures depend on the distance from some axis in the Lorentz-Minkowski plane $\mathbb{E}_{1}^{2}$ deserve to be studied as well. Work in this direction is in progress and will be reported elsewhere.

\section{References}

[1] Berger M. and Gostiaux B., Differential Geometry: Manifolds, Curves and Surfaces, Springer, New York 1988.

[2] Djondjorov P., Hadzhilazova M., Mladenov I. and Vassilev V., A Note on the Passage From the Free to the Elastica with a Tension, Geometry, Integrability and Quantization 10 (2009) 175-182.

[3] Ekmekci N. and Ilarslan K., On Bertrand Curves and Their Characterization, Differ. Geom. Dyn. Syst. 3 (2001) 17-24.

[4] Ferrández A., Giménez A. and Lucas P., Degenerate Curves in PseudoEuclidean Spaces of Index Two, Geometry, Integrability and Quantization 3 (2002) 209-223.

[5] Ferrández A., Giménez A. and Lucas P., Null Generalized Helices in LorentzMinkowski Spaces, J. Phys. A 35 (2002) 8243-8251. 
[6] İlarslan K., Some Special Curves on Non-Euclidian Manifolds, $\mathrm{PhD}$ Thesis, Graduate School of Natural and Applied Sciences, Ankara University 2002.

[7] Kuhnel W., Differential Geometry: Curves-Surfaces-Manifolds, Braunschweig, Wiesbaden 1999.

[8] Liu H. and Wang F., Mannheim Partner Curves in 3-Space, J. Geom. 88 (2008) 120-126.

[9] Lopez R., Differential Geometry of Curves and Surfaces in Lorentz-Minkowski Space, Int. Electron. J. Geom. 1 (2014) 44-107.

[10] Mladenov M., Hadzhilazova M., Djondjorov P. and Vassilev V., On the Generalized Sturmian Spirals, Comptes Rendus de l'Academie Bulgare des Sciences 64 (2011) 633-640.

[11] O'Neill B., Semi-Riemannian Geometry with Applications to Relativity, Academic Press, New York 1983.

[12] Opera J., Differential Geometry and Its Applications, Mathematical Association of America, Washington D.C. 2007.

[13] Singer A., Curves Whose Curvature Depends on Distance From the Origin, Am. Math. Montly 106 (1999) 835-541.

[14] Vassilev V., Djondjorov P. and Mladenov I., Integrable Dynamical Systems of the Frenet-Serret Type, In: Proc. 9th International Workshop on Complex Structures, Integrability and Vector Fields, World Scientific, Singapore 2009, pp 234-244.

[15] Zwikker C., The Advanced Geometry of Plane Curves and Their Applications, Dover, New York 1963.

Received 04 November 2014

Kazim İlarslan and Ali Uçum

Kırıkkale University

Faculty of Sciences and Arts

Department of Mathematics

Kirıkkale, TURKEY

E-mail address: kilarslan@yahoo.com

E-mail address: a liucum05@gmail.com

Ivaïlo M. Mladenov

Institute of Biophysics

Bulgarian Academy of Sciences

Acad. G. Bonchev Str., Block 21

1113 Sofia, BULGARIA

E-mail address: mladenov@bio21.bas.bg 